\title{
Recent advances in porous nanoparticles for drug delivery in antitumoral applications: inorganic nanoparticles and nanoscale metal-organic frameworks
}

\section{Alejandro Baeza, Daniel Ruiz-Molina \& María Vallet-Regí}

To cite this article: Alejandro Baeza, Daniel Ruiz-Molina \& María Vallet-Regí (2016): Recent advances in porous nanoparticles for drug delivery in antitumoral applications: inorganic nanoparticles and nanoscale metal-organic frameworks, Expert Opinion on Drug Delivery, DOI: 10.1080/17425247.2016.1229298

To link to this article: http://dx.doi.org/10.1080/17425247.2016.1229298

Accepted author version posted online: 30

Aug 2016.

Published online: 30 Aug 2016.

Submit your article to this journal $[\pi$

View related articles $\nearrow$

View Crossmark data ¿ 
Publisher: Taylor \& Francis

Journal: Expert Opinion on Drug Delivery

DOI: $10.1080 / 17425247.2016 .1229298$

Recent advances in porous nanoparticles for drug delivery in antitumoral applications: inorganic nanoparticles and nanoscale metal-organic frameworks

Alejandro Baeza, ${ }^{1,2} *$ Daniel Ruiz-Molina ${ }^{3}$ and María Vallet-Regí ${ }^{1,2}$

1. Center on Bioengineering, Biomaterials and Nanomedicine (CIBER-BBN), Spain

2. Departamento de Química Inorgánica y Bioinorgánica. Facultad de Farmacia, Universidad Complutense de Madrid. Instituto de Investigación Sanitaria Hospital 12 de Octubre i+12. Plaza Ramón y Cajal s/n, 28040 Madrid, Spain.

3. Catalan Institute of Nanoscience and Nanotechnology (ICN2), CSIC and The Barcelona Institute of Science and Technology, Campus UAB, Bellaterra, 08193 Barcelona, Spain

*Author for correspondence, email: abaezaga@ucm.es 


\section{ABSTRACT}

Introduction: Nanotechnology has provided new tools for addressing unmet clinical situations, especially in the oncology field. The development of smart nanocarriers able to deliver chemotherapeutic agents specifically to the diseased cells and to release them in a controlled way has offered a paramount advantage over conventional therapy.

Areas covered: Among the different types of nanoparticle that can be employed for this purpose, inorganic porous materials have received significant attention in the last decade due to their unique properties such as high loading capacity, chemical and physical robustness, low toxicity and easy and cheap production in the laboratory. This review discuss the recent advances performed in the application of porous inorganic and metal-organic materials for antitumoral therapy, paying special attention to the application of mesoporous silica, porous silicon and metal-organic nanoparticles.

Expert opinion: The use of porous inorganic nanoparticles as drug carriers for cancer therapy has the potential to improve the life expectancy of the patients affected by this disease. However, much work is needed to overcome their drawbacks, which are aggravated by their hard nature, exploiting the advantages offered by highly the ordered pore network of these materials.

Keywords: Cancer Nanomedicine, Porous Inorganic nanomaterials, Mesoporous silica nanoparticles, Porous silicon nanoparticles, Metal-Organic frameworks 


\section{Article highlights box}

- Porous inorganic nanocarriers present unique characteristics as consequence of their ordered pore network such as high loading capacity, ease and cheap synthesis, tunable morphology and controlled and sustained drug release profile, among others.

- Pore diameter in these materials can be adjusted within a broad range from $2 \mathrm{~nm}$, suitable for small drugs to a few tens of nanometers, useful for the transportation of therapeutic macromolecules.

- The external surface of these carriers can be decorated with different (bio)-moieties in order to provide targeting abilities against tumoral cells and/or stimuli-responsive behavior in the cargo release process.

- These materials present some liabilities in comparison with their organic counterparts such as a limited penetration in living tissues as a consequence of their rigid nature. Thus, the development of novel strategies which improve their penetration capacity is a critical point of their antitumoral efficacy. 


\section{INTRODUCTION}

In the recent years the scientific community has devoted huge efforts in the search of novel treatments against unmet clinical situations such as inoperable cancers, degenerative pathologies or resistant infections, among others. The application of nanotechnology to medicine, the so called nanomedicine, has become a key discipline for the discovery of novel therapies against these terrible diseases. This discipline comprises the use of nanometric systems as drug delivery carriers, medical imaging or sensors for clinical diagnostics, [1] being the first application the most studied with around $76 \%$ of the research papers in nanotechnology published in 2014. The utilization of nanoparticles as drug carriers provides several advantages such as improved pharmacokinetic profile, possibility to employ lipophilic drugs, higher circulation time in comparison with the administration of the free drugs and lower toxicity as a consequence of the lower dosages employed in the nanovehicles.[2] In 1986, the Japanese researchers Maeda and Matsumura reported that macromolecules bigger than $40 \mathrm{KDa}$ injected in the blood stream tended to accumulate preferably within tumoral tissues. They termed this phenomenon as enhanced permeation and retention (EPR effect).[3] This preliminary finding paved the way to the use of nanoparticles as drug delivery systems for antitumoral applications.[4] The reason for the accumulation of nanoparticles and big macromolecules into tumoral areas lies on the characteristic blood vessel architecture of tumoral tissues. Tumoral cells show a fast growing rate which requires high amounts of nutrients and oxygen. When the size of a tumoral mass exceeds $1 \mathrm{~mm}^{3}$ the diffusion of the nutrients is not enough to support the accelerated metabolism of the tumoral cells and they begin to suffer starvation. Thus, they force the surrounding healthy cells to produce proangiogenic factors which induce the creation of novel blood vessels. However, these novel vessels are not properly built, but they 
present pores and fenestrations with diameters up to a few hundreds of nanometers.[5] When the nanocarriers reach the tumoral tissue, they are able to pass through these fenestrations whereas they cannot pass through blood vessel epithelium present in healthy tissues. Additionally, the rapid growing rate of the tumoral cells usually compresses the lymphatic vessels compromising their normal function, which causes an inefficient drainage in the zone. The lack of a drainage system induces a higher retention of the nanoparticles extravasated in the tumoral mass. These two characteristics, higher permeability of the tumoral blood vessels (enhanced permeation) and lack of efficient lymphatic systems (enhanced retention) explain the preferential accumulation of the nanocarriers within the tumoral lesion. Almost 30 years later, a countless number of drug delivery nanosystems have been reported for oncological applications and even some of them have reached the market.[6] These nanocarriers can be formed by organic materials resulting in soft systems such as liposomes, polymersomes and micelles, among others, or can exhibit an inorganic nature originating tougher systems such as metallic or ceramic nanoparticles.[7] However, the use of pure inorganic materials in nanomedicine is strongly limited and it usually requires the creation of hybrid materials which conjugate the advantages of both type of systems.[8] Among the different materials that have been employed to build these hybrid nanocarriers, those systems which present ordered porosity constitute excellent materials for drug delivery applications due to their unique characteristics such as high loading capacity, chemical and physical robustness, low toxicity and easy and cheap production in the laboratory. The presence of the pore network increases considerably the available surface in comparison with solid counterparts. These pores can be engineered with a broad range of diameters, from 2 to a few tens of nanometers allowing the transportation of therapeutic agents with very different nature, such as 
small drugs, proteins, DNA or RNA strands and even other nanoparticles. Moreover, the ordered porosity of these materials allows more precise control over the drug cargo/departure process and also in the own material biodegradation. In this review, the recent advances carried out in the application of porous inorganic and metal-organic materials in drug delivery will be presented paying special attention to the application of mesoporous silica, porous silicon and metal-organic nanoparticles in antitumoral applications. For the synthesis of these materials, the reader could be referred to some excellent reviews.[9,10] Additionally, detailed descriptions of the different functionalization strategies for the decoration of nanomaterials with different (bio)moieties can be found elsewhere.[11]

\section{MESOPOROUS SILICA NANOPARTICLES}

Mesoporous silica nanoparticles (MSNs), specially presenting the MCM-41 structure, is a very promising material for drug delivery applications thanks to their specific properties. These nanoparticles can be easily obtained in the laboratory showing a wide range of morphologies, particle size and pore diameters.[12] MSNs presents high loading capacity $\left(600-1000 \mathrm{~m}^{2} \cdot \mathrm{g}^{-1}\right)$, high pore volume $\left(0.6-1 \mathrm{~mL} \cdot \mathrm{g}^{-1}\right)$ and pore size between $2-5 \mathrm{~nm}$. This last property allows the loading of species with very different nature, from small molecules to macromolecules such as proteins or DNA which can reach sizes of around a few dozens of nanometers. Additionally, the external and/or internal surface of these particles can be decorated with different functional groups such as amino, thiol or carboxylic groups, among others, using the correspondently functionalized alcoxysilanes through direct addition in one pot (co-condensation) or after the particle formation (post-synthesis). When a nanocarrier is employed for oncological applications, it is particularly important to avoid the premature release of the housed drugs due to the highly toxic nature which usually present these therapeutic 
agents. In the case of MSNs, it is possible to avoid the unwanted drug departure employing two different strategies. The first one consists in the attachment of different moieties (gatekeepers) on the pore outlets through covalent bonds which can be broken by an externally applied stimulus (light, magnetic fields, ultrasounds or temperature) or by an internal stimulus inherent to the treated pathology (acid or basic environments, change in redox conditions, presence of enzymes, etc.)[13] The second strategy for controlling the drug release consists in the coating of MSN surface with polymeric or lipidic shells which hampers the diffusion of the drugs trapped within the silica network.[14] These polymeric or lipid coatings are engineered to allow the drug release due to conformational changes in the polymeric layer once exposed to certain stimuli (Figure 1). In this section, the recent advances in the development of these stimuliresponsive materials carried out in the latest years will be briefly described. Due to the huge number of stimuli-responsive MSNs which have been reported, these devices will be separately described according to the stimulus employed for triggering the release. A more exhaustive description of this field has been reviewed elsewhere.[15]

\subsection{Light}

Light irradiation as triggering stimulus provides a precise control of the drug release location, being possible to apply the light beam in sub-millimetric regions. Contrariwise, its main liability is poor penetration in living tissues. Only light with wavelength located in the near infrared (NIR) window $(650-1350 \mathrm{~nm})$ is able to penetrate a few dozens of millimeters into a tissue. UV and, to a lesser extent, visible light, are strongly absorbed by living tissues and their use is limited to exposed or transparent regions. After the pioneering work of Fujiwara et al. who described the attachment of coumarin molecules on the pore entrances as UV-cleavable gatekeepers,[16] many different responsive MSNs which are triggered by UV light have 
been described.[17] Despite the poor penetration of UV light, the application of these devices could be possible for the treatment of exposed lesions such as skin, esophagus or colon tumors, or the light can be delivered to the target zone using a optic fiber. Lu et al. have reported the use of polymeric coatings on the surface of hollow MSN which can be degraded by the irradiation with green light $(540 \mathrm{~nm})$.[18] Additionally, folic acid was conjugated to the polymer branches in order to provide selectivity against tumoral cells which overexpress folate receptors showing excellent antitumoral response only under light irradiation. A modified azobenzene molecule able to suffer photoisomerization with red light $(625 \mathrm{~nm})$, which is more penetrating in living tissues, has been recently reported as light-sensitive gatekeeper.[19] The group of Thomas Bein has widely studied the attachment of photosensitizers (as protoporphyrin IX) on MSN surface in order to induce endosomal escape of these carriers when exposed to radiation at $405 \mathrm{~nm}$.[20] Nearly all the nanocarriers that are internalized by mammalian cells are uptaken via endocytosis. Therefore, to achieve rapid escape from the endosomes is of paramount importance for intracellular drug delivery in order to avoid the degradation of the transported molecules within late endosomes or lysosomes. Gold nanorods have been encapsulated within MSNs for inducing temperature increases in the surroundings after NIR exposition due to plasmonic photothermal conversion. Thus, Yang et al. employed calix[4]arenes which binds by supramolecular interactions quaternary ammonia groups placed on MSN that contains gold nanorods.[21] NIR irradiation produces hyperthermia in the region which reduces the binding affinity of the calix[4]arenes by the ammonia stalks causing the dissociation of these gatekeepers and the subsequent drug departure. Drug-loaded MSNs have been anchored on the surface of single-walled carbon nanotubes (SWNT) in order to combine the high loading capacity of MSN with the ability to transform NIR into thermal energy of SWNT.[22] 
This hybrid device is able to accumulate into tumoral lesions in a mice model and once there, to release its payload under NIR exposition acting simultaneously as contrast agent for photoacoustic imaging. Other approaches which exploit NIR-to-visible upconversion phenomena[23] and even X-rays[24] have been recently reported providing more strategies to the use of light as triggering stimulus.

\subsection{Temperature, magnetic field and ultrasounds}

There are different pathological conditions which are associated with a temperature increase. A general approach to achieve temperature-responsive behavior consists in coating the external surface of MSN with thermosensitive polymers, mainly based in $\operatorname{poly}(N$-isopropylacrylamide) (PNIPAM). This polymer suffers a conformational change from linear to globular state when the temperature exceeds $32{ }^{\circ} \mathrm{C}$. A polymeric shell placed on the external surface of MSNs act as a diffusion barrier which avoids premature release of the housed drugs when the temperature is kept below this value. However, if the temperature is higher, the polymer shell collapses and the retained drugs are able to escape from the silica matrix. This transition temperature is not useful for in vivo applications but it can be tuned to higher values between $40-45{ }^{\circ} \mathrm{C}$ adding hydrophilic monomers such as acrylamide, acrylic acid or $N$-hydroxymethylacrylamide to the polymer composition. Significant temperature increases can be achieved under alternative magnetic fields exposition of MSNs containing superparamagnetic iron oxide nanoparticles (SPIONs) trapped inside. Zink et al. have reported that the temperature of the particle surroundings can be increased almost $20^{\circ} \mathrm{C}$ after a short time magnetic field exposition, depending on the size of the SPIONs trapped within the silica matrix.[25] Thus, different engineered polymer coatings have been employed in combination with magnetic MSNs in order to control the release of both drugs trapped inside the pore network[26] and also higher macromolecules trapped within the polymer 
branches.[27] Moreover, magnetic MSN have been coated with lipid bilayers which are spontaneously disrupted at high temperatures.[28] Finally, DNA or peptide motifs have also been used as temperature responsive gatekeepers. [29]

Recently, Paris et al. have described the use of ultrasounds as triggering stimulus for drug release.[30] In this case, MSN surface was coated with a thermosensitive polymer which contains 2-tetrahydropyranyl methacrylate (THPMA) as monomer sensitive to ultrasounds. This polymer collapses at physiological temperature $\left(37^{\circ} \mathrm{C}\right)$ sealing the pore entrances, thus avoiding drug leakage. Under ultrasound irradiation, the acetal group of THPMA is broken enhancing the hydrophillicity of the polymer shell which places the transition temperature above this temperature and therefore, produces the pore opening.

\section{3. $p H$}

A nanoparticle which is travelling through the body will experience significant $\mathrm{pH}$ variations, from neutral $\mathrm{pH}$ in the blood stream to mild acidic conditions ( $\mathrm{pH}$ 5.5-6) in tumoral, inflamed and infected tissues. Additionally, the $\mathrm{pH}$ inside the cells is generally more acidic than the $\mathrm{pH}$ present in the intracellular media, especially in endosomes and lysosomes. Therefore, it is possible to exploit this fact in order to design nanocarriers able to release their payloads when they reach these intracellular organelles. One well established strategy to synthesize $\mathrm{pH}$-sensitive MSN is to coat the MSN external surface with polymers which present neutral charge at physiological $\mathrm{pH}$, whereas they become positively charged under mild-acidic conditions.[31] The dense polymer layer hampers the drug release at neutral $\mathrm{pH}$ but if $\mathrm{pH}$ drops to certain values, the repulsion forces exerted between charged chains distort the polymer layer allowing the drug departure. Another approach is the use of pore blockers which are attached on the pore outlets 
through sensitive bonds which are broken at mild-acidic conditions. Cai et al. have recently reported the development of hollow MSNs which exhibit a cascade process triggered by $\mathrm{pH}$.[32] The particle surface is functionalized with beta-cyclodextrins ( $\beta$ CDs) through boronate bonds. $\beta$-CDs bind to adamantane groups attached to PEG chains through imine bonds producing the pore closure. When the particle reaches the tumoral area $(\mathrm{pH}=6.8)$, the imine bonds are broken causing the PEG detachment which enhance the particle internalization by the tumoral cells. Then, as a consequence of the more acidic environment within endosomes $(\mathrm{pH}=4.5-6.5)$ boronate bonds suffer cleavage triggering the drug release. $\beta$-CDs bound to MSN surface by boronate bonds have been employed for the fabrication of MSNs responsive not only to $\mathrm{pH}$ variations but also to the presence of fructose exploiting the affinity of vincinal diols present in sugar moieties with the boronate group.[33] Lu et al. have described the use of small lanthanide nanoparticles as pore blockers which are bound to the pore outlets through acid-sensitive acetal bonds.[34] This device is able to release cytotoxic compounds loaded inside the pore network, acting at the same time as magnetic resonance imaging (MRI) contrast agent thanks to the presence of the rare-earth oxides. Zink et al. have recently described a MSN device able to release antibiotics in a $\mathrm{pH}$-triggered manner in order to destroy bacteria (F. tularensis) infecting human cells.[35] The pore closure/opening mechanism is based on supramolecular interactions between anilinoalkane attached on MSN surface and different cyclodextrins. Villegas et al. have recently published the decoration of the external surface of MSN with $\mathrm{pH}$ sensitive polymeric nanocapsules which contain collagenase trapped within their structure.[36] Thus, when the nanocarrier reaches the tumoral area, the acidic environment present in the tissue causes the disintegration of the polymeric capsule releasing collagenase which starts the degradation of the tumoral extracellular matrix. Therefore, an enhanced 
penetration of the nanocarrier is achieved which is of paramount importance in order to accomplish a homogeneous distribution of the nanomedicine along the diseased tissue (Figure 2).

\subsection{Redox}

The intracellular media is enriched in reductive species such as glutathione (GSH) in comparison with the extracellular environment. One approach to exploit this imbalance for triggering drug release is to attach polymers or pore blockers on the particle surface using dithiol bonds (S-S) which are broken by the presence of GSH.[37] Additionally, the polymer itself can be formed using redox-sensitive monomers or crosslinkers.[38] Shi et al. have synthesized hollow MSNs with large pores ( $24 \mathrm{~nm}$ of diameter, average) decorated with poly ( $\beta$-amino esters) through dithiol bonds in order to deliver, in a redox responsive manner, siRNA and doxorubicin, simultaneously.[39] Kim et al. have described the use of specific Fmoc-functionalized peptide chains which contain a S-S bond as redox-sensitive pore blocker.[40] These peptides adopt a specific turn-like conformation when attached on the pore entrance, avoiding the drug release. The addition of GSH produces a conformational change to a random structure allowing the retained drug to leak out from the particle. Finally, in a very recent work, De Cola et al. have reported a very interesting approach which consists in the preparation of MSNs containing breakable S-S- bonds within the silica framework in such a way that they are able to undergo accelerated degradation when exposed to the reductive intracellular media.[41]

\subsection{Macro- and small-molecules}

There are different pathologies in which certain enzymes or molecules are produced more abundantly than in the healthy state. In the case of oncology, tumoral cells of solid 
tumors usually overexpress proteolytic enzymes as metalloproteinases (MMP) or cathepsins in order to degrade the extracellular matrix and colonize other tissues. Avidin has been anchored on MSN surface by a biotynilated peptide chain which contains a sequence sensitive to MMP-9.[42] This device has been able to deliver two therapeutic agents (cisplatin and proteasome inhibitor bortezomib) specifically to tumoral cells in ex vivo $3 \mathrm{D}$ lung tissue cultures without affecting the healthy cells also present in the tumoral mass. Zhu et al. have recently described a very interesting MSN device able to release its payload in the presence of micro-RNA (miR-21) which is overexpressed by several tumoral cells.[43] This system is composed by MSN which contains quantum dots housed within the silica matrix in order to allow its traceability by fluorescence microscopy. The external surface is decorated with specific DNA strands which bind, by complementarity, hybrid DNA strands which contain the anti-miR-21 sequence and the AS1411 aptamer, this last in charge of the recognition of the tumoral cells. This hybrid structure acts as reversible pore blocker and targeting agent at the same time and it can be uncapped by the exposition to miR-21. Additionally, the presence or the higher concentration of small molecules or ions in pathological tissues can be exploited for triggering drug release. Au nanoparticles, as removable caps, have been anchored to the pore outlets using $\mathrm{Cu}^{2+}$ complexes as binding ion.[44] These copper complexes are broken at low $\mathrm{pH}$ values $(<5)$ and also in the presence of higher concentration of ATP $(7 \mathrm{mM})$.

\section{POROUS SILICON NANOPARTICLES}

Porous silicon nanoparticles (PSiNP) are composed by crystalline silicon crossed by multitude of pores with diameters comprised between 5-20 nm. Similarly to MSNs mentioned in the previous section, PSiNP present interesting properties for drug delivery applications such as high loading capacity (external surface of $200-500 \mathrm{~m} 2 \cdot \mathrm{g}^{-1}$ 
and pore volume of $0.5-2 \mathrm{~cm}^{3} \cdot \mathrm{g}^{-1}$ ) excellent biocompatibility, biodegradability and nonimmunogenic nature. Additionally, there are wide number of strategies for surface functionalization with different (bio)-moieties which provide targeting or stealth capacities in living hosts. Unlike MSNs which are synthesized using bottom-up approaches, PSiNP are generally produced by top-bottom strategies such as etching (chemical, laser-induced, metal-assisted, chemical vapor, etc.) and milling.[45] The PSiNP surface can be chemically modified previous or after the nanoparticle formation by different techniques as oxidation, carbonization and hydrosilylation. PSiNP are generally loaded by capillarity once the particles are immersed into a concentrated solution of the compound to be trapped. Positively charged molecules are more retained in this material than neutral or negative molecules due to the intrinsic negative charge of PSiNP. Additionally, the drugs or cargo molecules can be covalently attached in the pore walls. The attached drugs are released to the surrounding media once the material is degraded, or due to the rupture of the covalent bonds which bind the drug on the pore walls. Other option to retain molecules within the pore network consists in the oxidation of PSiNP after the loading procedure. Thus, the pore openings are closed which retain the drugs trapped as a consequence of the volume expansion caused by the oxygen incorporation. [46] Finally, when PSiNP are administered into a living organism is degraded to silicic acid, which is a harmless compound. PSiNP which contains large pores are rapidly degraded ( 8 hours) in $\mathrm{PBS}$ at $\mathrm{pH}=7.2$. However, this time is higher in the case of PSiNP with small pores $(<10 \mathrm{~nm})$ or it can be extended by external functionalization with different groups or polymers as PEG. In this section, some representative advances carried out with PSiNP will be briefly described in order to provide a panoramic picture of the power of this material for drug delivery applications. 
Antitumoral drugs have been loaded within the pore matrix of PSiNP producing nanocarriers able to destroy tumoral cells. Xia et al. have reported the fabrication of PSiNP functionalized with styrene groups in order to retain high amounts of doxorubicin $\left(660 \mu \mathrm{g} \cdot \mathrm{mg}^{-1}\right)$ by $\pi-\pi$ stacking between the aromatic rings of styrene and doxorubicin (Dox), respectively. The same research group has employed bovine serum albumin grafted on the particle surface in order to increase the colloidal stability of the system and also for loading Dox by electrostatic interactions. [47] Both systems are able to release the retained Dox at mild-acidic $\mathrm{pH}$. RNA interference encapsulated within small liposomes $(30-40 \mathrm{~nm})$ has been trapped within PSiNP in order to silence oncoproteins which play key role in tumoral progression.[48] The administration of one single injection of this system in a murine model of ovarian cancer was capable, not only to reduce the tumor burden, but also to hamper angiogenesis and tumoral cell proliferation without observing toxicity in the host. Voelker et al. have reported the use of PSi nanodiscs decorated with antibodies as targeted antitumoral nanocarriers.[49] In this work, MLR2 anti-p75 antibodies were grafted on the particle surface enhancing its selectivity for neuroblastoma cells (SH-SY5Y) which overexpress p75NTR neurotrophin receptors. These targeted nanodiscs were loaded with camptothecin showing high cytotoxicity and selectivity against the tumoral cells. Ferrari et al. has employed an engineered thioaptamer able to recognize E-selectin for achieving selective homing of PSi microparticles to bone marrow.[50] The employed thioaptamer specifically binds to E-selectin, which is usually expressed by the bone marrow endothelium, whereas it shows little affinity by other selectin family members. The surface of PSiNP can be decorated not only with targeting molecules but also with imaging agents giving place to theranostics devices, i.e. nanocarriers able to deliver therapeutic compounds and to provide information by imaging techniques about their 
biodistribution or therapeutic efficacy in real time. In a recent paper, a theranostics PSiNP has been synthesized placing iRGD as targeting agent and ${ }^{111}$ In-DOTA and Alexa-Fluor 488 as single photon emission computed tomography (SPEC) and fluorescent agent, respectively.[51] This device combines the enhanced selectivity against metastatic prostate cancer provided by iRGD, a dual-modality imaging capacity and a controlled antitumoral drug release. Polymeric coatings can be placed on the particle surface in order to control the release kinetic of the housed drugs. Chitosan coating has been employed with this purpose on the surface of oligonucleotide-loaded PSiNP.[52] The positive charge of this polymer promotes its adhesion by electrostatic interactions on the negatively charged particle surface. Additionally, the resulting particle presents a positive surface which facilitates the interaction with cell membranes and therefore, the particle uptake. In the case of naked particles, the retained oligonucleotides are rapidly released ( $80 \%$ of the cargo is released in the first 4 hours) whereas it requires more than 35 hours when the particles are coated.

PSiNP can be loaded with two or more species, even with molecules which present very different nature, in order to combine several therapeutic effects, or to beat the acquired drug resistance of tumoral cells (Figure 3).

Thus, indomethacin, a hydrophobic anti-inflammatory drug and hydrophilic peptides as PYY3-36, a 36 aminoacids peptide that inhibit the appetite, were effectively loaded within PSiNP showing acceleration in their release profile and higher drug permeation in tissue models as a consequence of their mutual influence.[53] In other recent work, methotrexate, a folic acid analog which is used as antitumoral drug was chemically grafted on amino-functionalized PSiNP walls and sorafenib, an anti-angiogenic hydrophobic drug, was loaded within the pore network.[54] This material shown fast release of the hydrophobic drug thanks to the highly porous nature of the carrier and 
prolonged release of methotrexate, as a consequence to the need to break the covalent bond which maintains this molecule attached to the surface. This particular release kinetic could be exploited for achieving a rapid angiogenesis inhibition followed by tumoral cell death. Weitz et al. have reported an assembled nanocarrier composed by PSiNP embedded within giant liposomes. This device is able to deliver different species such as hydrophilic and hydrophobic drugs, DNA nanostructures, gold nanorods and iron oxide nanoparticles.[55] The presence of the metallic cores provides responsive capacities under photothermal and magnetic exposition because the heat generated by the application of these stimuli distorts the lipid bilayer. The combined release of different cytotoxic drugs in combination with the release of certain DNA nanostructures engineered to enhance the action of the antitumoral drugs have demonstrated the capacity to destroy multi-drug resistance breast cancer cells, which are resilient to the administration of these cytotoxic drugs alone.

These types of particles are uptaked by tumoral cells through endocytosis in a similar way that MSNs and the vast majority of nanocarriers. Thus, endosomal escape is of paramount importance in order to reach the cytosol avoiding the aggressive environment usually present in late endosomes or lysosomes. This fact is even more dramatic in the case of the transportation of labile molecules as proteins, DNA, RNA, etc. Santos et al. have reported the use of a zwitterionic polymer coating composed by polyethylene imine (PEI) and poly(methyl vinyl ether-co-maleic acid) (PMVE-MA).

This polymer shell provides several interesting advantages such as increased colloidal stability caused by electrostatic repulsion between the particles, endosomal escape as a consequence of proton sponge effect originated by the presence of tertiary amines in PEI and finally, a sustained drug release behavior due to the polymer coating.[56] 
Finally, similarly to the case of MSN although much less exploited, different stimuliresponsive gatekeepers can be anchored on the pore outlets in order to control drug departure. Thus, cyclodextrins have been anchored on the pore entrances using supramolecular interactions which can be broken at mild acidic conditions.[57] $\mathrm{pH}-$ sensitive polymers as poly(beta-amino esters) have also been employed as gate-keepers in this type of material. [58]

\section{METAL-ORGANIC FRAMEWORKS (MOFs)}

Coordination polymers (CPs) have emerged as a novel family of nanostructured materials for encapsulation and drug release applications over the last years.[59] Advantages, if properly addressed, are multifold: (i) nanoscale CPs show intrinsic benefits associated with its hybrid nature, i.e. the combination of metal ions and organic ligands; (ii) they exhibit a high synthetic flexibility as long as metal-ligand bonds exhibit directional interactions that can be used to systematically control and tune their dimensionality; (iii) CPs have magnetic, electronic, optical, and catalytic properties associated with the limitless choice of metallic elements they can contain and (iv) different metal elements haye ubiquitous functions in natural biological systems. Therefore, although CPs in crystalline forms date are known for many years, their miniaturization to the nanometer scale has represented a novel opportunity to develop a unique class of highly tailorable functional materials that combine the rich diversity of CPs with the advantages of nanomaterials.

Two different approaches schematically represented in Figure 4 have already been followed for the encapsulation and controlled release of antitumoral drugs:[60] I) amorphous coordination polymer nanoparticles, refereed from now on as nanoscale 
coordination polymers (NCPs)[61] and II) nanoscale crystalline and porous coordination polymer structures, referred from now on as NMOFs

Although nanoscale NCPs do not exhibit an open-framework structure, they have already shown great potential for encapsulation of different drugs with yields up to $20 \%$ [62] and improved IC50 values with respect to the corresponding free drug [63]. In these systems, encapsulation takes place through a physical entrapment of the drug within the amorphous polymeric internal structure of the nanoparticles. However, much better encapsulation yields and modified release profiles can be obtained by incorporating the drugs as constitutive units of the coordination network in the form of active ligands or with connecting metal ions such as Pt(IV) complexes.[64]

On the contrary, NMOFs exhibit tunable pores with an exceptionally high surface area and high loading capacities. [65] Their geometries, size, and functionalities can be systematically varied to yield architecturally robust porous structures with a typical porosity up to $50 \%$ of the crystal volume. These materials exhibit a wide range in pore diameter between $2-50 \mathrm{~nm}$, similarly to the materials described above. Accordingly surface areas can range from 1000 to $10,000 \mathrm{~m}^{2} / \mathrm{g}$, much higher than those of other traditional porous materials such as zeolites and carbons. Therefore, encapsulation usually takes place within the pores though some authors such as Monti et al. have demonstrated that drug encapsulation can also be mediated through the reactivity of the drug with the metal ions of the framework.[66] For this, MIL-100(Fe) nanostructures of $\sim 200 \mathrm{~nm}$ diameter were loaded with DOX and the binding constants determined via absorption and fluorescence titrations. Spectroscopic data indicated that DOX binding occurs via the formation of highly stable coordination bonds between one or both deprotonated hydroxyl groups of the aglycone moiety and coordinatively unsaturated Fe(III) centers. Alternatively, other area of interest within the field is the 
functionalization of the NMOFs to improve their colloidal stability and biocompatibility. In this direction, Bein et al. have demonstrated that MOF@lipid systems can effectively store dye molecules inside their porous scaffold while the addition of a protective lipid bilayer: I) prevents their premature release, II) increases the colloidal stability of the nanoparticles and III) favors a high uptake of lipid coated nanoparticles by cancer cells (Figure 5).[67]

Finally, as far as the loading of active drugs within MOFs is concerned, the group of Ferey is considered one of the pioneering groups. Back in 2006, they already demonstrated that MIL-101 (Cr) can adsorb $138 \mathrm{wt} \%$ ibuprofen and MIL-53 can adsorb 22 wt \% ibuprofen. The release of ibuprofen from the MILs was evaluated using simulated body fluid at $37{ }^{\circ} \mathrm{C}$. It was found that the MIL-101 (Cr) released ibuprofen slowly in several stages, reaching completion after 6 days.[68] The MIL-53 materials showed an even slower release, reaching completion after 21 days.[69] Lin et al. have also been pioneers to show the potential application of NMOFs in magnetic resonance imaging and anticancer drug delivery applications.[70]

As previously described, NMOFs have already been demonstrated to be excellent carriers for drug delivery applications. Among the different therapeutic areas of interest, recent advances in the development of NMOFs for drug delivery have been specifically focalized in antitumoral applications, which are summarized next.

As far as the encapsulation of antitumoral drugs is concerned, Li et al. reported back in 2009 the synthesis of the MIL-101 NMOF and its loading with an organic fluorophore and an anticancer drug via covalent modifications of the as-synthesized nanoparticles.[71] Afterwards, Horcajada, Greft et al.[72] encapsulated antitumoural and retroviral drugs such as busulfan, azidothymidine triphosphate, doxorubicin or 
cidofovir within non-toxic porous iron(III)-based metal-organic frameworks with high loadings. Beyond the encapsulation process, these authors also demonstrated the potential association of therapeutics and diagnostics. Since then, the number of examples describing the potential use of NMOFs with antitumoral applications has considerably increased, as summarized next.

\subsection{Single reports of small drugs}

Wong et al. have reported the loading of a dinuclear gold(I) pyrrolidinedithiocarbamato complex within a $\mathrm{Zn}^{2+}$-based metal-organic framework (Zn-MOF) with in vitro cytotoxic activities towards A2780cis cisplatin-resistant ovarian cancer cells. Interestingly, drug-release testing was done using a set of transwell assay-based experiments instead of the conventional dialysis approach.[73] Zr-based UiO-66 cubical nanostructures with average diameters of $70 \mathrm{~nm}$ were also reported by Shi et al. to load Alendronate (AL), a bisphosphate anticancer drug. Cytotoxicity assays in HepG2 and MCF-7 cells showed that these nanostructures enhance cell killing by comparison with free AL.[74] Alternatively, Wang et al. developed $\mathrm{Zn}^{2+}$ or $\mathrm{Cu}^{2+} \mathrm{NMOFs}$, using the cytotoxic ligand, 3,5-bis(pyridine-3-ylmethylamino)benzoic acid, which exhibited cytotoxicity effects in three human cancer cells (NCI-H446, MCF-7 and HeLa).[75]

An enjoyable example of the advantages of drug encapsulation was given by Gref at el. [76] These authors encapsulated the highly hydrophilic prodrug phosphated gemcitabin (Gem-MP), known for its instability and inability to bypass cell membranes, within MIL-100 NMOFs. Interestingly, the storage stability of the loaded NMOFs was strongly dependent on the media; indeed, while the NMOF turns out to be stable in water at least for three days significant release was found in media containing phosphates since it induces its particle degradation. Moreover, the drug-loaded NMOFs were effective 
against pancreatic PANC-1 cells in contrast to free drug and empty NMOFs, which apparently did not show any cytotoxic effect.

Finally, multifunctional systems combining anticancer activity and imaging capacities have also been a target of interest. With this aim Sahu et al. have incorporated $\mathrm{Fe}_{3} \mathrm{O}_{4}$ nanoparticles, used as an MRI contrast agents, into nanostructures smaller than $100 \mathrm{~nm}$ of the porous isoreticular IRMOF-3.[77] Such nanostructures were subsequently conjugated with folic acid (to achieve targeted drug delivery towards cancer cells) and the fluorescent rhodamine B isothiocyanate (RITC) (for biological imaging), and loaded with the hydrophobic anticancer drug paclitaxel. In vitro biological toxicity studies revealed that the resulting nanoparticles targeted and killed the cancer cells in a highly effective manner.

\subsection{Encapsulation and controlled release of most common drugs}

\subsubsection{Camptothecin and Doxorubicin}

In a recent work crystals of the well-known ZIF-8 were loaded with Doxorubicin (DOX) and used as efficient drug delivery carriers with efficacies on breast cancer cell lines remarkably higher than those found for free DOX.[78] Yamauchi et al. used the same family of ZIF-8 NMOFs to encapsulate $0.049 \mathrm{~g}$ DOX/g ZIF-8; in this case, cytotoxicity studies against three different human cancer cells (NCI-H292, HT29 and HL-60) resulted in a moderate activity by comparison with free DOX.[79] Moderate cytotoxicity versus leukemia cell line U937 was also found for Gd-based nanostructures of $\sim 140 \mathrm{~nm}$ obtained upon mechanical downsize from bulk MOFs via ball milling and encapsulating up to $12 \mathrm{wt} \%$ of DOX.[80] In a further work, NMOFs of the MIL-101 loading doxo were developed by a one-pot synthesis and its premature drug release controlled upon surface modification with a $\mathrm{pH}$ responsive benzoic imine bond and a 
redox active disulfide system. Accordingly, in vitro and in vivo results demonstrated how this system exhibited effective cancer cell inhibition while having reduced side effects.[81]

\subsubsection{Methotrexate}

Gd-BDC nanorods coated with Methotrexate (MTX), PNIPAM-co-PNAOSco-PFMAMTX, and further linked with a targeting ligand GRGDS- $\mathrm{NH}_{2}$ were shown to exhibit enhanced cytotoxicity in sarcoma cells FITZ-HAS as compared to nontargeted NMOFs. Moreover, and thanks to the presence of the gadolinium ions, the nanoorods have simultaneous MRI activity.[82] MTX has also been used as a bridging ligand in $\mathrm{NMOF}$ combined with $\mathrm{Zn}^{2+}$ or $\mathrm{Gd}^{3+}$ metal ions, resulting in astonishing high drug loadings (up to $79 \mathrm{wt} \%$ ). The spherical nanostructures, with diameter ranging from 40 to $100 \mathrm{~nm}$, were stabilized with a lipid bilayer and targeted with anisamide. Efficient cellular uptake was confirmed by confocal microscopy studies though cytotoxicity studies revealed a behavior comparable to that of free drug.[83] Qian et al. also encapsulated MTX with high yields into inner pores and channels of the porphyrinbased MOF PCN-221 by diffusion and controlled its posterior release under physiological environment without "burst effect".[84] While the empty MOF framework exhibited low cytotoxic effects on the PC12 cells, the controlled pH release of the corresponding loaded nanoparticles revealed its activity in oral drug delivery.

\subsubsection{5-Fluorouracil}

This is without any doubt one of the antitumoral drugs most widely loaded within NMOFs, as confirmed by the numerous examples so far reported. For instance, very recently Yang et al. have described microporous UiO-66- $\mathrm{NH}_{2}$ particles loading the drug 
and [2]pseudorotaxanes as gates of the nanocarriers linked via host-guest complexation to regulate the drug-controlled release.[85]

More emphasis has been given to Zn-based NMOFs. Wang et al. used zinc and the hexadentate ligand 5,5',5'-(1,3,5-triazine-2,4,6-triyl)tris(azanediyl)triisophthalate (TATAT) to prepare a chiral nanoporous MOF with high porosity. Afterwards, the antitumoral drug 5-Fu was loaded with high yield (about $50 \%$ of the transported drug versus the carrier material) and shown to be slowly released with a complete delivery time of about one week.[86] Other authors have reported a $\mathrm{Zn}_{3}(\mathrm{~L}) \mathrm{MOF}$ that can accommodate up to $0.36 \mathrm{mg}$ of 5 -Fu per $\mathrm{mg}$ MOF combined with lanthanide (III) cations for luminescence applications.[87] Loading of 5-Fu in into the well-known ZIF8 NMOFs have also been reported on different examples. For instance, Wang et al. reported control over the loading of 5-Fu into ZIF-8 NMOFs with a amount up to $\sim 40$ wt $\%$ and the posterior release under a $\mathrm{pH}$ sensitive environment.[88] Lan et al. also demonstrated that ZIF-8 MOFs could load 5-Fu with high loading amounts (31 wt \%) and 5-Fu could diffuse out of the framework without burst.[89] Wang et al. showed that ZIF-8 NMOFs with a diameter of 100-200 nm by TEM could carry both 5-Fu and green fluorescent $\mathrm{C}$-dots for $\mathrm{pH}-$-responsive drug release and fluorescence imaging.[90] Other authors developed three polyoxometalates (POMs) and loaded the POMs into ZIF-8 NMOFs with an average size of 50-200 $\mathrm{nm}$.[91] The authors demonstrated that the incorporation of POMs into the frameworks led to more efficient loadings of 5-Fu and slow release of 5-Fu from the particles. Zhang et al. have very recently studied the biocompatibility and biodistribution of fluorouracil loaded ZIF-8 nanoparticles (ZIFNPs). A surprising high concentration was found in lung though the drug levels drop dramatically with time, revealing the fast degradation and elimination of these nanosystems. Accordingly, at the given doses, ZIF-NPs exhibit reasonably biosafety in 
animal tests as evidenced by their acceptable system and blood biocompatibilities, and minimal impacts on the liver and renal functions, immune cells, inflammatory factors, etc. However, ZIF-NPs with fluorouracil loading (5Fu@ZIF-NPs) significantly improve the therapeutic outcome of lung metastasis tumor in a nude mice model.[92]

Finally some examples of copper-based MOFs have been reported. For example, $\mathrm{Ng}$ et al. reported MOFs with a formula of $\left[\mathrm{Cu}(\mathrm{L})\left(4,4^{\prime}-\right.\right.$ bipy $\left.)\left(\mathrm{H}_{2} \mathrm{O}\right)\right]$, where $\mathrm{H}_{2} \mathrm{~L}$ stands for diphenylmethane-4,4'-dicarboxylic acid, loaded with amounts of 5-Fu up to $28 \mathrm{wt}$ \%.[93] Nascimento and co-workers reported the synthesis of a Cu-BTC MOF incorporating the 5-Fu drug with yields of $\sim 45 \mathrm{wt} \%$.[94] The cytotoxicity of drug loaded MOFs was evaluated in different cell lines (NCI-H292, MCF-7, HT29 and HL60) showing enhanced cytotoxicity mainly in MCF-7 and HL60 cells. In a further example, Zhou et al. synthesized a Cu-based MOF with conjugated PEG5K on the surface via click chemistry resulting in PEGylated MOF nanoparticles of $\sim 50 \mathrm{~nm}$ that allow for the controlled loading and release of the drug.[95]

\subsubsection{Platinum and Ruthenium}

NMOFs based on the assembly of $\mathrm{Zn}^{2+}$ metal ions and a functionalized pyrazol-based organic spacer have been recently reported by Barea et al.[96] These system were shown to exhibit excellent colloidal stabilities under different relevant intravenous and oral-simulated physiological conditions, fact that the authors attributed to the formation of a protein corona on their surface. Furthermore, two antitumor drugs (mitroxantrone and $\left[\mathrm{Ru}(\mathrm{p}\right.$-cymene $) \mathrm{Cl}_{2}$ (pta) $]$ (RAPTA-C) where pta =1,3,5-triaza-7-phospaadamantane) were encapsulated with a loading capacity that directly depends on the surface area of the solids and its functionalization. 
Multifunctionality has also been explored by Morris et al. upon combined loading of platinum and ruthenium drugs with NO. For instance, incorporation of cisplatin and a $\mathrm{Pt}(\mathrm{IV})$ cisplatin prodrug into two zirconium-based UiO66 and UiO66- $\mathrm{NH}_{2}$ MOFs following two different approaches has been reported.[97] In the first route, the $\mathrm{Pt}(\mathrm{IV})$ cisplatin prodrug was incorporated into $\mathrm{UiO} 66-\mathrm{NH}_{2}$ through an amide coupling reaction with the $\mathrm{NH}_{2}$ groups whereas in the second route, cisplatin was encapsulated into the large cavities of both MOFs. The cytotoxicity of the formulations was assessed on the A549 lung cancer cell line showing that the cisplatin loaded MOF turns out to be more efficient because its higher loading capacity. The same authors also investigated the multifunctionality of these systems by incorporation of the antithrombotic NO into the drug-loaded MOFs; surprisingly, the amount of NO released from these formulations is much greater than that from the pure MOFs. Morris and co-workers further loaded not only platinum but also the chemotherapeutic agent, $\left[\mathrm{Ru}\left(\mathrm{p}\right.\right.$-cymene) $\left.\mathrm{Cl}_{2}(\mathrm{pta})\right]$ (RAPTAC), along with NO into the same MOF by direct interaction with the Ni open metal sites and physically entrapment, respectively. The loading efficiency of NO and RAPTA-C was not affected by the presence of each other. However, the presence of RAPTA-C in the MOFs significantly retarded the desorption of NO under a humid flowing gas. MIL88(Fe) MOFs were also exploited as the delivery vehicle for NO by Morris and coworkers. A significant amount of NO was adsorbed at room temperature by the nontoxic, biodegradable, and flexible MIL-88(Fe) MOFs at a high loading amount of 1- $-2.5 \mathrm{mmol} / \mathrm{g}$. NO was released from MOFs over a long period of time $(>16 \mathrm{~h})$, suggesting these MOFs can adsorb NO with high efficiency and release NO in a controlled manner.[98]

Multifunctionality has also been reported with other systems beyond NO. For example, Lin and co-workers reported the first use of NMOFs for the combined delivery of 
cisplatin and pooled siRNA.[99] For this, cisplatin and a pool of siRNAs targeting multidrug resistant genes were loaded into UiO hexagonal plate like NMOFs with $\sim 100$ $\mathrm{nm}$ diameter and $\sim 30 \mathrm{~nm}$ thickness with high loading amounts. Interestingly, these systems efficiently delivered both siRNA and cisplatin to four cisplatin-resistant human ovarian cancer cells (ES-2, OVCAR-3, SKOV-3 and A2780/CDDP) decreasing the cisplatin IC50 values by an order of magnitude as compared to free cisplatin. This efficacy increase was attributed to the activation of the drug resistant gene from the ovarian cancer cells to cisplatin mediated by siRNA/UiO-Cis NMOFs.

\section{CONCLUSIONS}

Even though porous materials are still far from becoming a real and widely approved solution for drug delivery into the market, these materials have already shown their versatility and efficiency in many crucial areas of relevance, from high yield encapsulation to targeted cytotoxicity. Comparatively, most of the work has been done on mesoporous silica nanoparticles by comparison with the two other approximations having some prototypes in clinical trials.

First reports on the use of PSiNP and NMOFs for antitumoral applications were described less than a decade ago but since then the number of citations and papers being reported in the area is increasing exponentially. Much of the work so far devoted is focussed on fundamental concepts of increasing loading encapsulation yields, surface functionalization and the interaction of drug with different carriers. Though, work in the near future should be concentrated more on their colloidal stability, permeability and drug release responses in real biological environments.

\section{EXPERT OPINION}




\subsection{General Comments}

Porous nanoparticles constitute one of the most promising materials for clinical use in the treatment of cancer. Advantages are multifold: I) they exhibit a great loading capacity clearly higher than the capacity shown by other organic systems as polymersomes, micelles, liposomes, or definitely over non-porous inorganic materials, II) trapped drugs can be released in a controlled manner both placing stimuliresponsive gatekeepers on their external surface as well as modifying the inner pore walls in order to release the cargo under certain conditions and HI) the solid nature of the carrier provides high protection to the trapped species against the aggression of external agents. Most of the successful examples so far reported with hybrid porous materials for drug delivery in antitumoral applications are based on mesoporous silica nanoparticles and porous silicon to a lesser extent. On the contrary, though successful examples have already been reported, much work is needed for NMOFs. Efforts in this direction are already being undertaken through the introduction of a large number of different functional groups within the pores of MOFs. This yields multivariate frameworks in which the varying arrangement of functionalities gives rise to materials that offer a synergistic combination of properties. Another area to be studied with more detail in NMOFs is their biodistribution and accumulation, including cellular transit, degradation, excretion, physiological barrier penetration, and chronic toxicity. In vivo studies of the pharmacokinetics and efficiency of drug-containing NMOFs should be the next major steps to evaluate their real performance in medicine. Nevertheless, and albeit

yet at relative early stages of development, we do believe that the precise chemical control that is possible to attain over the assembly of NMOFs will definitely propel this field further into new realms of synthetic chemistry in which far more sophisticated materials may be accessed. For example, materials can be envisaged as having (i) 
compartments linked together to operate separately, yet function synergistically; (ii) dexterity to carry out parallel operations; (iii) ability to count, sort, and code information; and (iv)capability of dynamics with high fidelity.

\subsection{Size and shape}

Another area of relevance is that of the final dimensions of the nanostructures. Indeed, the collapse of lymphatic vessels within a tumor facilitates the retention of extravasated nanoparticles in the tumoral area; nevertheless, this also causes the apparition of interstitial fluid pressure which strongly hampers their diffusion within the zone. Therefore, the action of these nanomedicines is restricted to the periphery of the tumors which drastically reduces their therapeutic efficacy. As a general rule to overcome this limitation, smaller sizes of nanocarrier achieve deeper penetrations. However, higher sizes yield better discrimination between healthy and tumoral tissues. Therefore, it is necessary to optimize the size carrier for each type of tumor. MSNs can be easily prepared with a wide range a shapes, sizes and external functionalization.[12] The influence of these parameters in cell uptake processes have been widely studied showing that the aspect ratio and surface charge play an important role in cell internalization and this process is highly cell dependent.[100] Additionally, in the case of intravenous administration (that is the usual case for antitumoral therapy) it is necessary to take into account the influence of these parameters in the interaction of the nanocarriers with the blood cells. However, in most cases these parameters have been eyaluated using simplistic in vitro models and much work is required in order to know their significance in animal models. NMOFs with good dimensions between tens of nanometers and a few hundred nanometers have been reported though with some irregular shapes in some cases, plate-like structures or not perfectly round-shaped nanoparticles. Therefore detailed studies about the effect of the shape would be 
required. Another limitation of nanocarrier diffusion is caused by the high collagen density present in tumoral tissues. In order to improve the diffusion, proteolytic enzymes have been administered a few days before nanoparticle administration, or even these enzymes have been anchored on the particle surface.[36] In the case of porous nanoparticles, their lack of flexibility may difficult even more the penetration in living tissues and the barriers mentioned above play an even more important role. Thus, it is compulsory to take into account these barriers in order to design a suitable nanocarrier for future developments.

\subsection{Surface functionalization}

Another area of relevance lies at the surface functionalization of the nanoparticles and their effect on their improved stabilities and cell internalization. Indeed, one of the main advantages of nanoparticles as drug carriers for oncological applications is their passive accumulation within tumoral lesions by EPR effect. Once there, the presence of targeting agents attached on the particle surface enhances their uptake in these diseased cells, without affecting the healthy ones which are also present in the tissue. This fact allows the selective destruction of malignant cells. However, despite the good outcomes observed in in vitro experiments, only a few targeted nanodevices have demonstrated good performance in animal models. There are several reasons which contribute to this failure. One of them is that when a nanocarrier comes into contact with blood, it is immediately covered by a protein layer called protein corona. This corona masks the targeting agents which are the real readable part of the nanocarrier. Thus, the fate of the

nanocarrier is not ruled by the presence of the targeting agents on the surface but it depends on the proteins bound to the particle surface. Additionally, the presence of the protein corona usually accelerates the particle clearance by the mononuclear phagocytic system (MPS). These limitations can be alleviated grafting the targeting agents 
employing hydrophilic or zwitterionic polymers as spacers. These polymers avoid protein adsorption enhancing the circulating time and they also present more effectively the targeting agents to tumoral cell receptors. Another issue which limits the in vivo nanocarrier performance is the high complexity and heterogeneity of the tumoral mass which not only present several types of tumoral cells but it also contains a huge number of non-tumoral cells as mesenchymal, immune, supportive cells, etc. which play an important role in tumor progression. In many cases, the efficacy of targeted nanodevices is tested employing simplified in vitro models which contain only the tumoral cell. It is true that in vitro models provides valuable information about the recognition process between the targeting agent and the specific tumoral cell receptor but, before reaching in vivo evaluation, it should be necessary to evaluate this capacity employing more realistic models such as $3 \mathrm{D}$ tissue models or tumor spheroids which contains a representative mixture of tumoral and non-tumoral cells, as well as cells from the immune systems. Finally, another drawback associated with the use of targeted nanomedicines is the binding site barrier, which is caused by the strong affinity between the targeting moiety and its receptor. This fact provokes that nanomedicines are firmly bound to the first cell line near tumoral blood vessels exacerbating even more the poor penetration problem mentioned above. One way to reduce this effect is to employ stimuli-responsive targeting agents which travel through the body in a hidden conformation, whereas they can be activated by certain stimulus present in the tumoral area or externally applied.[101] Great success has been achieved on the surface functionalization of silica nanoparticles. A high number of synthetic strategies for the decoration of the external surface of MSN and PSiNP have been described. It is even possible to functionalize differently the external surface and inner pore walls. However, in many cases it is necessary to achieve a precise control of the orientation of the grafted 
biomolecule, as in the case of antibodies or other recognition moieties. In this sense, it would be very useful to employ the wide arsenal of synthetic alternatives which provides the bioorthogonal chemistry.[102] Once more, surface functionalization is less common for NMOFs most of the times limited to the reactivity of some metal ions lying at the surface of the nanostructures or mainly to some polymeric or lipidic coatings.

Another limitation, not only for porous nanoparticles but common to several nanocarriers, lies in the fact that passive accumulation by EPR effect is not always guaranteed because this phenomenon is not universal. It not only depends on the tumor type but it also exhibits great heterogeneity within one tumoral lesion. Additionally, EPR undergoes variations during the treatment. Thus, tumors which exhibit a significant EPR effect at the beginning of the treatment could show scarce passive accumulation after some cycles of treatment. Therefore, it is necessary to monitor the vascular architecture of the tumoral lesion before recommending a treatment based on nanomedicines. It is possible to enhance the EPR effect through the previous administration of drugs as angiotensin I-converting enzyme (ACE) inhibitors or NO releasing agents.[4]

It is important to keep in mind that the design of a nanocarrier able to overcome all of these barriers would be pointless, because it would require to build really complex systems, difficult to approve by regulatory agencies.[103] The administration of diverse types of nanocarriers, each of them responsible for different tasks, could be simpler and more efficient than the design of one single carrier able to perform all of them. There are a few examples of cooperative work between nanoparticles in which, for example one of them facilitates the extravasation of the second one in the tumoral area,[104] but more efforts are needed in this point. As was pointed out above, there are many parameters which affect the efficacy of these types of nanocarriers and more knowledge 
is needed in order to find solutions for them. For the design of the next generation of novel porous inorganic nanocarriers we have to bear in mind the well-known quote in the engineering world "embrace complexity, design versatility and deliver simplicity". 


\section{Funding}

The authors acknowledge financial support from Spanish Government through the project MAT2015-64831-R and MAT2015-70615-R and by FEDER funds. D RuizMolina thanks ICN2-CSIC, Spain for infrastructural and financial support and specially the students and collaborators for their contribution. ICN2 acknowledges support from the Severo Ochoa Program (MINECO, Grant SEV-2013-0295). M Vallet-Regíis grateful thanks the financial support through ERC-2015-AdG VERDI.

Declaration of interest

The authors have no relevant affiliations or financial involvement with any organization or entity with a financial interest in or financial conflict with the subject matter or materials discussed in the manuscript. This includes employment, consultancies, honoraria, stock ownership or options, expert testimony, grants or patents received or pending, or royalties. 


\section{References}

\section{Papers of special note have been highlighted as either of interest (•) or of considerable interest $(\bullet \bullet)$ to readers}

1. Tibbitt MW, Dahlman JE, Langer R. Emerging frontiers in drug delivery. J Am Chem Soc 2016;138:704-17.

2. Brigger I, Dubernet C, Couvreur P. Nanoparticles in cancer therapy and diagnosis. Ady Drug Deliv Rev 2012;64:24-36.

3. Matsumura Y, Maeda H. A new concept for macromolecular therapeutics in cancerchemotherapy - mechanism of tumoritropic accumulation of proteins and the antitumor agent smancs. Cancer Res 1986;46:6387-92.

4. Maeda H, Nakamura H, Fang J. The EPR effect for macromolecular drug delivery to solid tumors: Improvement of tumor uptake, lowering of systemic toxicity, and distinct tumor imaging in vivo. Adv Drug Deliv Rev 2013;65:71-79.

5. Jain RK, Stylianopoulos T. Delivering nanomedicine to solid tumors. Nat Rev Clin Oncol 2010;7:653-64.

- Review of the principal mechanisms of EPR effect and the main limitations of the use of nanocarrier as antitumoral agents.

6. [10] Steichen SD, Caldorera-Moore M, Peppas N. A review of current nanoparticle and targeting moieties for the delivery of cancer therapeutics. Eur J Pharm Sci 2013;48:416-27.

7. Peer D, Karp JM, Hong S, et al. Nanocarriers as an emerging platform for cancer therapy. Nat Nanotechnol 2007;2:751-760.

8. Nguyen KT, Zhao Y. Engineered hybrid nanoparticles for on-demand diagnostics and therapeutics. Acc Chem Res 2015;48:3016-25.

9. Hoffmann F, Cornelius M, Morell J, et al. Silica-based mesoporous organic-inorganic hybrid materials. Angew Chem Int Ed 2006;45:3216-51.

$\bullet \bullet$ Review of the synthetic proccedures for the synthesis of MSNs and their mechanism.

10. Chudley R, Greeno R. Fundamentals of Porous Silicon Preparation. Porous Silicon Pract. Prep. Charact. Appl. 2012, Chapter 1:1-42.

11. Sapsford KE, Algar WR, Berti L, et al. Functionalizing nanoparticles with biological molecules: Developing chemistries that facilitate nanotechnology. Chem Rev 2013;113:19042074.

12. Trewyn BG, Slowing II, Giri S, et al. Synthesis and functionalization of a mesoporous silica nanoparticle based on the sol-gel process and applications in controlled release. Acc Chem Res 2007;40:846-53.

13. Yang P, Gaib S, Lin J. Functionalized mesoporous silica materials for controlled drug delivery. Chem Soc Rev 2012;41:3679-3698. 
14. Argyo C, Weiss V, Bräuchle C,et al. Multifunctional mesoporous silica nanoparticles as a universal platform for drug delivery. Chem Mater 2014;26:435-51

15. Baeza A, Colilla M, Vallet-Regí M. Advances in mesoporous silica nanoparticles for targeted stimuli-responsive drug delivery. Expert Opin Drug Deliv 2015;12:319-37.

16. Mal NK, Fujiwara M, Tanaka Y. Photocontrolled reversible release of guest molecules from coumarin-modified mesoporous silica. Nature 2003;421:350-53.

17. Martínez-Carmona M, Baeza A, Rodriguez-Milla M, etal. Mesoporous silica nanoparticles grafted with a light-responsive protein shell for highly cytotoxic antitumoral therapy. J Mater Chem B 2015;3:5746-52.

18. Yang $\mathrm{S}$, Li N, Chen $\mathrm{D}$, et al. Visible-light degradable polymer coated hollow mesoporous silica nanoparticles for controlled drug release and cell imaging. J Mater Chem B $2013 ; 1: 4628-36$.

19. Wang D, Wu S. Red-light-responsive supramolecular valves for photocontrolled drug release from mesoporous nanoparticles. Langmuir 2016;32:632-6

20. Dobay MP, Schmidt A, Mendoza E, et al. Cell type determines the light-induced endosomal escape kinetics of multifunctional mesoporous silica nanoparticles. Nano Lett 2013;13:1047-52.

21. Li H, Tan L-L, Jia P, et al. Near-infrared light-responsive supramolecular nanovalve based on mesoporous silica-coated gold nanorods. Chem Sci 2014;5:2804-8.

22. Liu J, Wang C, Wang X, et al. Mesoporous silica coated single-walled carbon nanotubes as a multifunctional light-responsive platform for cancer combination therapy. Adv Funct Mater 2015;25:384-92.

23. Xu F, Ding L, Tao W, et al. Mesoporous-silica-coated upconversion nanoparticles loaded with vitamin B12 for near-infrared-light mediated photodynamic therapy. Mater Lett 2016;167:205-8.

24. Chen $\mathrm{H}$, Wang GD, Chuang Y-J, et al. Nanoscintillator-mediated X-ray inducible photodynamic therapy for in vivo cancer treatment. Nano Lett 2015;15:2249-56.

25. Dong J, Zink JI. Taking the temperature of the interiors of magnetically heated nanoparticles. ACS Nano 2014;8:5199-207.

26. Guisasola E, Baeza A, Talelli M, et al. Magnetic-responsive release controlled by hot spot effect. Langmuir 2015;31:12777-82.

27. Baeza A, Guisasola E, Ruiz-Hernandez E, et al. Magnetically triggered multidrug release by hybrid mesoporous silica nanoparticles. Chem Mater 2012;24:517-24.

28. Bringas E, Koysuren O, Quach D V, et al. Triggered release in lipid bilayer-capped mesoporous silica nanoparticles containing SPION using an alternating magnetic field. Chem Commun 2012;48:5647-49. 
29. Ruiz-Hernandez E, Baeza A, Vallet-Regi M. Smart drug delivery through dna/magnetic nanoparticle gates. ACS Nano 2011;5:1259-66.

30. Paris JL, Cabanas MV, Manzano M, et al. Polymer-grafted mesoporous silica nanoparticles as ultrasound-responsive drug carriers. ACS Nano 2015;9:11023-33.

31. Niedermayer S, Weiss V, Herrmann A, et al. Multifunctional polymer-capped mesoporous silica nanoparticles for $\mathrm{pH}$-responsive targeted drug delivery. Nanoscale 2015;7:7953-64.

32. Liu J, Luo Z, Zhang J, et al. Hollow mesoporous silica nanoparticles facilitated drug delivery via cascade $\mathrm{pH}$ stimuli in tumor microenvironment for tumor therapy. Biomaterials 2016;83:51-65.

33. Qiu X-L, Li Q-L, Zhou Y, et al. Sugar and $\mathrm{pH}$ dual-responsive snap-top nanocarriers based on mesoporous silica-coated $\mathrm{Fe}_{3} \mathrm{O}_{4}$ magnetic nanoparticles for cargo delivery. Chem Commun 2015;51:4237-40.

34. Chen Y, Ai K, Liu J, et al. Multifunctional envelope-type mesoporous silica nanoparticles for $\mathrm{pH}$-responsive drug delivery and magnetic resonance imaging. Biomaterials 2015;60:111-20.

35. Li Z, Clemens DL, Lee BY, et al. Mesoporous silica nanoparticles with $\mathrm{pH}$-sensitive nanovalves for delivery of moxifloxacin provide improved treatment of lethal pneumonic tularemia. ACS Nano 2015;9:10778-89.

36. Villegas MR, Baeza A, Vallet Regí M. Hybrid collagenase nanocapsules for enhanced nanocarrier penetration in tumoral tissues. ACS Appl Mater Interfaces 2015;7:24075-81.

37. Prasad R, Aiyer S, Chauhan DS, et al. Bioresponsive carbon nano-gated multifunctional mesoporous silica for cancer theranostics. Nanoscale 2016;8:4537-46.

38. Palanikumar L, Choi ES, Cheon JY, et al. Noncovalent polymer-gatekeeper in mesoporous silica nanoparticles as a targeted drug delivery platform. Adv Funct Mater 2015;25:957-965.

39. Wu M, Meng Q, Chen Y, et al. Large pore-sized hollow mesoporous organosilica for redox-responsive gene delivery and synergistic cancer chemotherapy. Adv Mater 2016;28:19639.

40. Lee J, Kim H, Han S, et al. Stimuli-responsive conformational conversion of peptide gatekeepers for controlled release of guests from mesoporous silica nanocontainers. J Am Chem Soc 2014;136:12880-3.

41. Maggini L, Cabrera I, Ruiz-Carretero A, et al. Breakable mesoporous silica nanoparticles for targeted drug delivery. Nanoscale 2016;8:7240-7.

42. Tumors ML, Rijt SH Van, Bo Da, et al. Protease-mediated release of chemotherapeutics from mesoporous silica nanoparticles to ex vivo human. ACS Nano, 2015;9: 2377-89 
43. Zhang P, Cheng F, Zhou R, et al. DNA-hybrid-gated multifunctional mesoporous silica nanocarriers for dual-targeted and microRNA-responsive controlled drug delivery. Angew Chem Int Ed 2014;53:2371-5.

44. Chen X, Cheng X, Soeriyadi AH, et al. Stimuli-responsive functionalized mesoporous silica nanoparticles for drug release in response to various biological stimuli. Biomater Sci $2014 ; 2: 121$.

45. Santos HA, Mäkilä E, Airaksinen AJ, et al. Porous silicon nanoparticles for nanomedicine: preparation and biomedical applications. Nanomedicine 2014;9:535-54.

46. Anglin EJ, Cheng L, Freeman WR, et al. Porous silicon in drug delivery deyices and materials. Adv Drug Deliv Rev 2008;60:1266-77.

- Review of the synthetic procedures and applications of porous silicon in medicine.

47. Xia B, Zhang W, Shi J, et al. A novel strategy to fabricate doxorubicin/bovine serum albumin/porous silicon nanocomposites with $\mathrm{pH}$-triggered drug delivery for cancer therapy in vitro. J Mater Chem B 2014;2:5280.

48. Tanaka T, Mangala LS, Vivas-Mejia PE, et al. Sustained small interfering RNA delivery by mesoporous silicon particles. Cancer Res 2010;70:3687-96.

49. Alhmoud H, Delalat B, Elnathan R, et al. Porous silicon nanodiscs for targeted drug delivery. Adv Funct Mater 2015;25:1137-45.

50. Mann AP, Tanaka T, Somasunderam A, et al. E-selectin-targeted porous silicon particle for nanoparticle delivery to the bone marrow. Adv Mater 2011;23:278-82.

51. Wang C-FC-F, Sarparanta MP, Mäkilä EM, et al. Multifunctional porous silicon nanoparticles for cancer theranostics. Biomaterials 2015;48:108-18.

52. Kafshgari MH, Delalat B, Tong WY, et al. Oligonucleotide delivery by chitosanfunctionalized porous silicon nanoparticles. Nano Res 2015;8:2033-46.

53. Liu D, Bimbo LM, Mäkilä E, et al. Co-delivery of a hydrophobic small molecule and a hydrophilic peptide by porous silicon nanoparticles. J Control Release 2013;170:268-78.

54. Wang CF, Mäkilä EM, Kaasalainen MH, et al. Dual-drug delivery by porous silicon nanoparticles for improved cellular uptake, sustained release, and combination therapy. Acta Biomater 2015;16:206-14.

55. Kong $\mathrm{F}$, Zhang $\mathrm{X}$, Zhang $\mathrm{H}$, et al. Inhibition of multidrug resistance of cancer cells by co-delivery of dna nanostructures and drugs using porous silicon nanoparticles@giant liposomes. Adv Funct Mater 2015;25:3330-40.

56. Shahbazi MA, Almeida P V., Mäkilä EM, et al. Augmented cellular trafficking and endosomal escape of porous silicon nanoparticles via zwitterionic bilayer polymer surface engineering. Biomaterials 2014;35:7488-7500.

57. Xue M, Zhong X, Shaposhnik Z, et al. PH-operated mechanized porous silicon nanoparticles. J Am Chem Soc 2011;133:8798-801. 
58. Xu W, Thapa R, Liu D, et al. Smart porous silicon nanoparticles with polymeric coatings for sequential combination therapy. Mol. Pharmaceutics 2015;12:4038-47.

59. Novio F, Simmchen J, Vázquez-Mera N, et al. Coordination polymer nanoparticles in medicine. Coord Chem Rev 2013;257:2839-47.

60. He Ch, Liu D, Lin W. Nanomedicine applications of hybrid nanomaterials built from metal-ligand coordination bonds: nanoscale metal-organic frameworks and nanoscale coordination polymers. Chem Rev 2015;115:11079-108.

61. Della Rocca J, Liu D, Lin W. Nanoscale metal-organic frameworks for biomedical imaging and drug delivery. Acc Chem Res 2011;44:957-68.

62. Imaz I, Rubio-Martínez M, García-Fernández L, et al. Coordination polymer partícles as potential drug delivery systems. Chem Comm 2010;46:4737-9.

63. Novio F, Lorenzo J, Nador F, et al. Carboxyl group ([bond]co2h) functionalized coordination polymer nanoparticles as efficient platforms for drug delivery. Chem Eur $\mathrm{J}$ 2014;20:15443-50.

64. Rieter WJ, Pott KM, Taylor KML, et al. Nanoscale coordination polymers for platinumbased anticancer drug delivery. J Am Chem Soc 2008;130:11584-5.

65. Horcajada P, Serre C, Vallet-Regí M, et al. Metal-organic frameworks as efficient materials for drug delivery. Angew. Chem. 2006;118:6120-4.

66. Anand R, Borghi F, Manoli F, et al. Host-guest interactions in $\mathrm{Fe}(\mathrm{III})$-trimesate MOF nanoparticles loaded with doxorubicin. J Phys Chem B 2014;118:8532-9.

67. Wuttke S, Braig S, Preiß TS, et al. MOF nanoparticles coated by lipid bilayers and their uptake by cancer cells. Chem Commun 2015;51:15752-5.

68. Horcajada P, Serre C, Vallet-Regí M, et al. Metal-organic frameworks as efficient materials for drug delivery. Angew Chem Int Ed 2006;45:5974-8.

69. Horcajada P, Serre C, Maurin G, et al. Flexible porous metal-organic frameworks for a controlled drug delivery. J Am Chem Soc 2008;130:6774-80.

70. Taylor KML, Rieter WJ, Lin W. Manganese-based nanoscale metal-organic frameworks for magnetic resonance imaging. J Am Chem Soc 2008;130:14358-9.

71. Taylor-Pashow KML, Della Rocca J, Xie Z, et al. Postsynthetic modifications of ironcarboxylate nanoscale metal-organic frameworks for imaging and drug delivery. J Am Chem Soc 2009;131:14261-3.

72. Horcajada P, Chalati T, Serre C, et al. Porous metal-organic-framework nanoscale carriers as a potential platform for drug delivery and imaging. Nat Mat 2010;9:172-8.

73. Sun RWY, Zhang M, Li D, et al. Dinuclear gold(I) pyrrolidinedithiocarbamato complex: cytotoxic and antimigratory activities on cancer cells and the use of metal-organic framework. Chem Eur J 2015;21:18534-8. 
74. Zhu XY, Gu JL, Wang Y, et al. Inherent anchorages in UiO-66 nanoparticles for efficient capture of alendronate and its mediated release. Chem Commun 2014;50:8779-82.

75. Wang KB, Ma XY, Shao DL, et al. Coordination-induced assembly of coordination polymer submicrospheres: promising antibacterial and in vitro anticancer activities. Cryst Growth Des 2012;12:3786-91.

76. Rodriguez-Ruiz V, Maksimenko A, Anand R, et al. Efficient "green" encapsulation of a highly hydrophilic anticancer drug in metal-organic framework nanoparticles. J Drug Target 2015;23:759-67.

77. Chowdhuri AR, Bhattacharyab D, Sahu SK. Magnetic nanoscale metal organic frameworks for potential targeted anticancer drug delivery, imaging and as an MRI contrast agent. Dalton Trans 2016;45:2963-73.

78. Zheng H, Zhang Y, Liu L, et al. One-pot synthesis of metal-organic frameworks with encapsulated target molecules and their applications for controlled drug delivery. J Am Chem Soc 2016;138:962-8.

79. Torad NL, Li YQ, Ishihara S, et al. MOF-derived nanoporous carbon as intracellular drug delivery carriers. Chem Lett 2014;43:717-9.

80. Kundu T, Mitra S, Patra P, et al. Mechanical downsizing of a gadolinium(iii)-based metal-organic framework for anticancer drug delivery. Chem Eur J 2014;20:10514-8.

81. Wang XG, Dong ZY, Cheng H, et al. A multifunctional metal-organic framework based tumor targeting drug delivery system for cancer therapy. Nanoscale 2015;7:16061-70.

82. Rowe MD, Thamm DH, Kraft SL, et al. Polymer-modified gadolinium metal-organic framework nanoparticles used as multifunctional nanomedicines for the targeted imaging and treatment of cancer. Biomacromolecules 2009;10:983-93.

83. Huxford RC, Dekrafft KE, Boyle WS, et al. Lipid-coated nanoscale coordination polymers for targeted delivery of antifolates to cancer cells. Chem Sci 2012;3:198-204.

84. Lin W, Hu Q, Jiang K, et al. J Sol State Chem 2016;237:307-12.

85. Tan LD, $\mathrm{Li} \mathrm{H}, \mathrm{Zhou} \mathrm{Y}$, et al. $\mathrm{Zn}^{2+}$-Triggered drug release from biocompatible zirconium mofs equipped with supramolecular gates. Small 2015;11:3807-13.

86. Sun CY, Qin C, Wang C, et al. Chiral nanoporous metal-organic frameworks with high porosity as materials for drug delivery. Adv Mater 2011;23:5629-32.

87. Wang Y, Yang J, Liu YY, et al. Controllable syntheses of porous metal-organic frameworks: encapsulation of LnIII cations for tunable luminescence and small drug molecules for efficient delivery. Chem - Eur J 2013;19:14591-9.

88. Sun CY, Qin C, Wang XL, et al. Zeolitic imidazolate framework-8 as efficient pHsensitive drug delivery vehicle. Dalton Trans 2012;41:6906-9. 
89. Qin JS, Du DY, Li WL, et al. N-rich zeolite-like metal-organic framework with sodalite topology: high $\mathrm{CO} 2$ uptake, selective gas adsorption and efficient drug delivery. Chem Sci 2012;3:2114-8.

90. He L, Wang TT, An JP, et al. Carbon nanodots@zeolitic imidazolate framework-8 nanoparticles for simultaneous $\mathrm{pH}$-responsive drug delivery and fluorescence imaging. Cryst Eng Comm 2014;16:3259-63.

91. Li R, Ren X, Zhao J, et al. Polyoxometallates trapped in a zeolitic imidazolate framework leading to high uptake and selectivity of bioactive molecules. J Mater Chem A 2014;2:2168-73.

92. Li S, Wang K, Shi Y, et al. Novel biological functions of ZIF-NP as a delivery vehicle: high pulmonary accumulation, favorable biocompatibility, and improved therapeutic outcome. Adv Funct Mater 2016;26:2715-27.

93. Liu JQ, Wu J, Jia ZB, et al. Two isoreticular metal-organic frameworks with CdSO4like topology: selective gas sorption and drug delivery. Dalton Trans 2014:43:17265-73.

94. Lucena FR, de Araujo LC, Rodrigues Mdo D, et al. Induction of cancer cell death by apoptosis and slow release of 5-fluoracil from metal-organic frameworks $\mathrm{Cu}$-BTC. Biomed Pharmacother 2013;67:707-13.

95. Zhao D, Tan SW, Yuan DQ, et al. Surface functionalization of porous coordination nanocages via click chemistry and their application in drug delivery. Adv Mater 2011;23:90-93.

96. Rojas S, Carmona FJ, Maldonado CR, et al. Nanoscaled zinc pyrazolate metal-organic frameworks as drug-delivery systems. Inorg Chem 2016;55:2650-63.

97. Mocniak KA, Kubajewska I, Spillane DEM, et al. Incorporation of cisplatin into the metal-organic frameworks UiO66-NH2 and UiO66-encapsulation vs. conjugation. RSC Adv 2015;5:83648-56.

98. McKinlay AC, Eubank JF, Wuttke S, et al. Nitric oxide adsorption and delivery in flexible mil-88(fe) metal-organic frameworks. Chem Mater 2013;25:1592-9.

99. He C, Lu K, Liu D, et al. Nanoscale metal-organic frameworks for the co-delivery of cisplatin and pooled sirnas to enhance therapeutic efficacy in drug-resistant ovarian cancer cells. J Am Chem Soc 2014;136:5181-4.

100. Huang $\mathrm{X}$, Teng $\mathrm{X}$, Chen $\mathrm{D}$, et al. The effect of the shape of mesoporous silica nanoparticles on cellular uptake and cell function. Biomaterials 2010;31:438-48.

101. Du J, Lane LA, Nie S. Stimuli-responsive nanoparticles for targeting the tumor microenvironment. J Control Release 2015;219:205-14.

102. Algar WR, Prasuhn DE, Stewart MH, et al. The controlled display of biomolecules on nanoparticles: A challenge suited to bioorthogonal chemistry. Bioconjug Chem 2011;22:825-58.

- Review of the orthogonal chemical strategies for anchoring biomolecules on nanoparticles. 
103. Venditto VJ, Szoka Jr. FC. Cancer nanomedicines: So many papers and so few drugs! Adv Drug Deliv Rev 2013;65:80-8.

104. Park J-H, von Maltzahn G, Xu MJ, et al. Cooperative nanomaterial system to sensitize, target, and treat tumors. Proc Natl Acad Sci USA 2010;107:981-6.

\section{FIGURES}
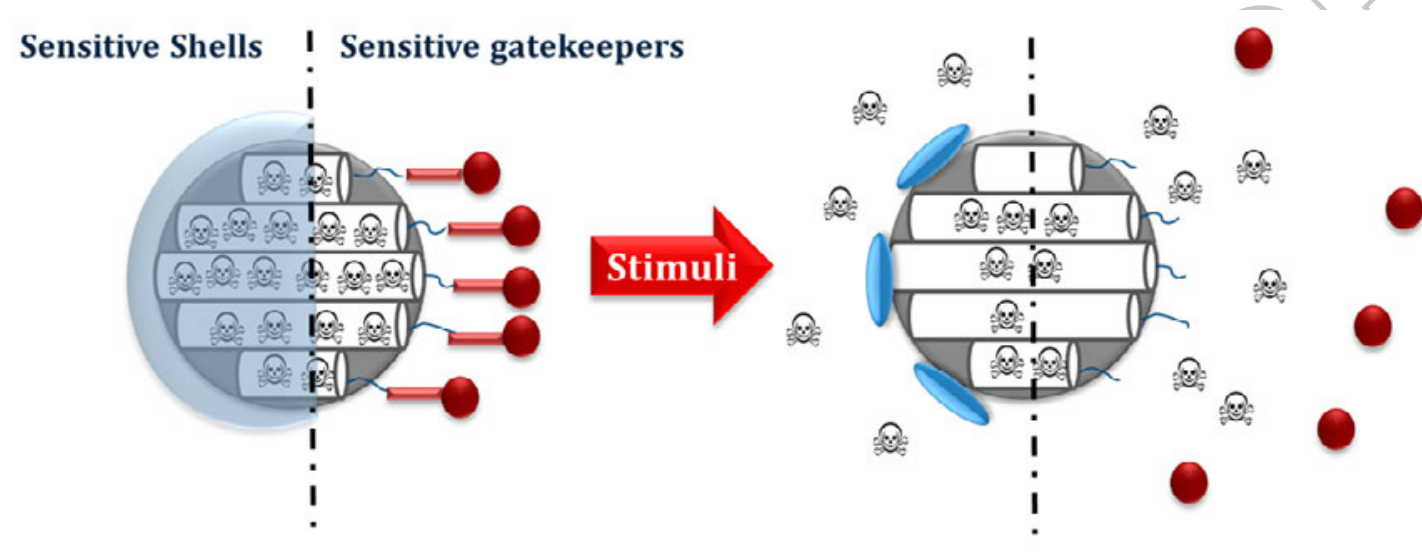

Figure 1. Strategies for controlled release in MSNS 


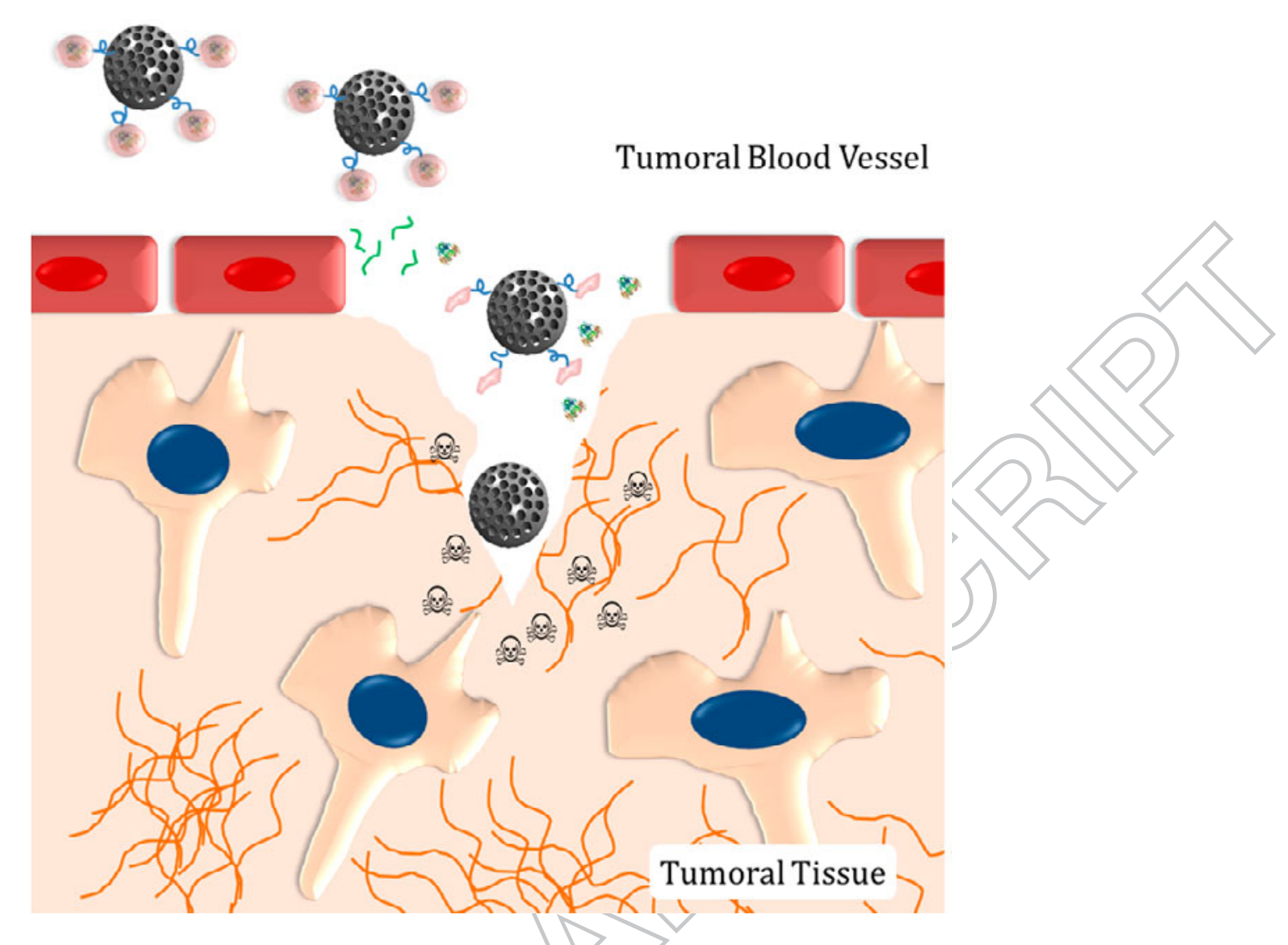

Figure 2. pH-sensitive collagenase nanocapsules grafted on MSNs for improved penetration in tumoral tissues 


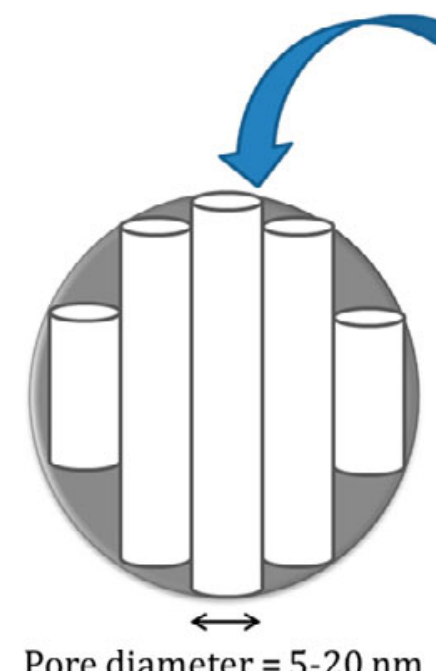

\section{Drugs}

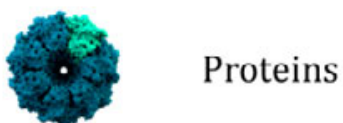

4

D Oligonucleotides (DNA or RNA)

Pore diameter $=5-20 \mathrm{~nm}$

Small Nanoparticles

Figure 3. Multidrug delivery capacity in PSiNP 

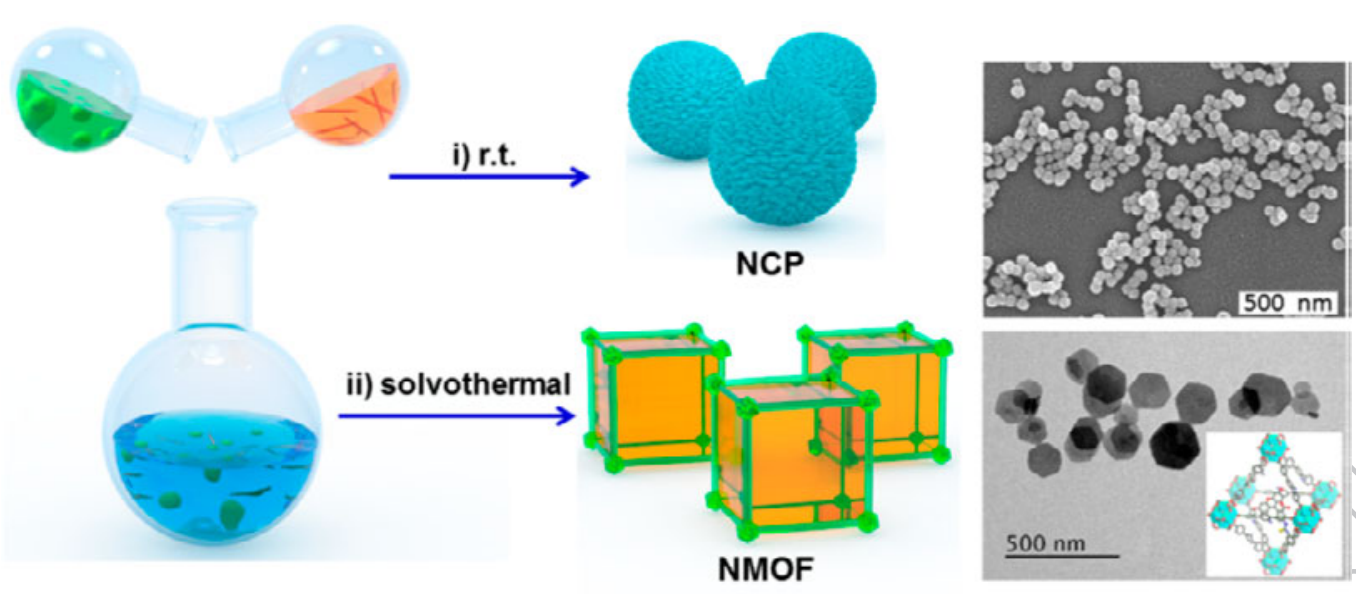

Figure 4. Schematic representation of the two different approaches followed with coordination polymers for their use as drug carriers.

Reprinted with permission from $\mathrm{He} \mathrm{Ch}$, Liu D, Lin W. Nanomedicine applications of hybrid nanomaterials built from metal-ligand coordination bonds: nanoscale metalorganic frameworks and nanoscale coordination polymers. Chem Rev 2015;115:11079-108. Copyright 2016 American Chemical Society. 


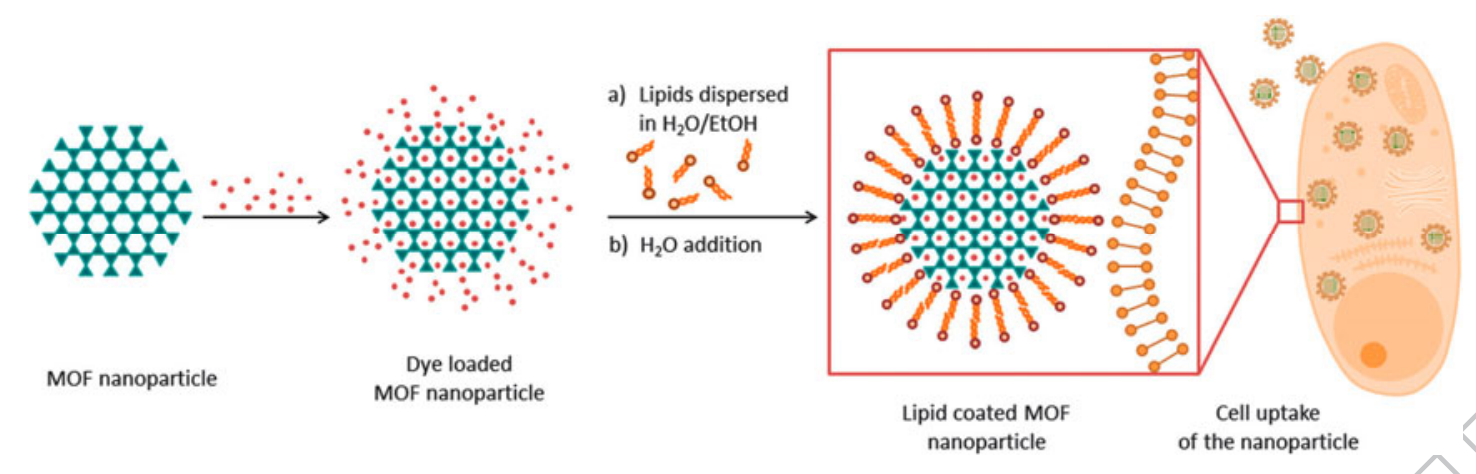

Figure 5. Schematic description of the synthesis of lipid bilayer-coated MOF nanoparticles loaded with dye molecules and their uptake in cancer cells.

Reproduced with permission from S. Wuttke, S. Braig, T. Preiß, A. Zimpel, J. Sicklinger, C. Bellomo, J. O. Rädler, A. M. Vollmar and T. Bein, Chem. Commun., 2015, 51, 15752 - Published by The Royal Society of Chemistry. 